\title{
Phase-conjugate reflection by degenerate four-wave mixing in a nematic liquid crystal in the isotropic phase
}

\author{
D. Fekete, J. AuYeung, and A. Yariv \\ California Institute of Technology, Pasadena, California 91125
}

Received October 8, 1979

\begin{abstract}
We report the generation of conjugate wave fronts by degenerate four-wave mixing in the isotropic phase of the nematic substance $p$-methoxy-benzylidene $p$ - $n$-butylaniline. The temporal and spatial properties of the conjugate wave fronts are verified. The dependence of the nonlinear reflectivity on the pump-wave power and the temperature of the medium is discussed.
\end{abstract}

Liquid-crystal materials are composed of molecules with strong anisotropy. An optical field can induce appreciable molecular alignment along the field, resulting in a change of the index of refraction that is proportional to the optical intensity. Third-order nonlinear optical phenomena, such as self-focusing of light, ${ }^{1,2}$ stimulated scattering, ${ }^{3}$ and third-harmonic generation, ${ }^{4-6}$ have been observed in these materials. In this Letter, the generation of phase-conjugate wave fronts by degenerate four-wave mixing in $p$-methoxybenzylidene $p$ - $n$-butylaniline (MBBA) is reported. MBBA is a nematic liquid crystal at room temperature. The experiment, however, utilized the isotropic phase of this material, which manifests some field-induced short-range order above the transition temperature, $T_{c}$. The nonlinear optical properties of MBBA in this pretransitional state have been investigated elsewhere, ${ }^{7-9}$ and this material has been found to possess a large nonlinear optical susceptibility, about an order of magnitude larger than that of carbon disulfide.

In the presence of an applied optical field with components $E_{k}(\omega)$, the third-order nonlinear polarization in an isotropic medium is ${ }^{9}$

$$
\begin{aligned}
P_{i}^{(3)}(\omega)= & \sum_{j} 6\left[\chi_{i i j j}^{(3)} E_{i}(\omega) E_{j}(\omega) E_{j}^{*}(\omega)\right. \\
& +\chi_{i j i j}^{(3)} E_{j}(\omega) E_{i}(\omega) E_{j}^{*}(\omega) \\
& \left.+\chi_{i j j i}^{(3)} E_{j}(\omega) E_{j}(\omega) E_{i}^{*}(\omega)\right] .
\end{aligned}
$$

The nonlinear susceptibility tensor $\chi_{i j k l}$ is made up of two parts; for example, $\chi_{\mathrm{ijj}}^{(3)}$ is typically written as

$$
\chi_{i j j i}^{(3)}=A+B .
$$

$A$ is an electronic contribution that is due to the optical-field-induced deformation of the electron cloud around the molecules. $B$ is the nuclear part that is due to the molecular response to the applied fields. It has been found by Shen et al. ${ }^{9}$ that the electronic contribution to the third-order nonlinear susceptibility in MBBA is negligible and that the dominant susceptibility tensor element is $\chi_{i j j i}^{(3)}$, which, in this case, is equal to $B$. Unlike the electrons, which can respond instantaneously to the applied fields, the nucleus responds more slowly and has a longer relaxation time. The re- laxation time of the nuclear response for MBBA varies inversely with temperature, ranging from $800 \mathrm{nsec}$ near the transition temperature $T_{c}$ to about $40 \mathrm{nsec}$ at $10^{\circ} \mathrm{C}$ above $T_{c} \cdot{ }^{9}$

In the degenerate four-wave mixing process, the nonlinear medium is illuminated by two intense pump fields, both at a frequency $\omega$. These fields are chosen to be plane waves,

$$
\begin{aligned}
& \mathbf{E}_{1}(\mathbf{r}, \mathbf{t})=A_{1}(t) \exp \left[i\left(\omega \mathbf{t}-\mathbf{k}_{1} \cdot \mathbf{r}\right)\right] \hat{y}, \\
& \mathbf{E}_{2}(\mathbf{r}, \mathbf{t})=A_{2}(t) \exp \left[i\left(\omega t-\mathbf{k}_{2} \cdot \mathbf{r}\right)\right] \hat{y},
\end{aligned}
$$

which are counterpropagating, so $\mathbf{k}_{1}(\omega)+\mathbf{k}_{2}(\omega)=0$. Upon incidence, along an arbitrary direction of an arbitrary input wave,

$$
\mathbf{E}_{4}(\mathbf{r}, \mathbf{t})=A_{4}(\mathbf{r}, \mathbf{t}) \exp \left[i\left(\omega t-\mathbf{k}_{\mathbf{4}} \cdot \mathbf{r}\right)\right] \hat{x}
$$

at the same frequency $\omega$ but with a wave vector $\mathbf{k}_{4}(\omega)$, a third-order nonlinear polarization is induced in the MBBA and is of the form

$$
\begin{aligned}
\mathbf{P}_{\mathrm{NL}}(\mathbf{r}, \mathbf{t})=6 \frac{\mathrm{B}}{\tau}\left\{E_{2}(\mathbf{r}, \mathbf{t})\right. & \int_{-\infty}^{t} \exp \left[-\left(t-t^{\prime}\right) / \tau\right] \\
\times E_{1}\left(\mathbf{r}, \mathbf{t}^{\prime}\right) E_{4}^{*}\left(\mathbf{r}, \mathbf{t}^{\prime}\right) \mathrm{d} t^{\prime}+ & E_{1}(\mathbf{r}, \mathbf{t}) \int_{-\infty}^{t} \exp \left[-\left(t-t^{\prime}\right) / \tau\right] \\
& \left.\times E_{2}\left(\mathbf{r}, \mathbf{t}^{\prime}\right) E_{4}^{*}\left(\mathbf{r}, \mathbf{t}^{\prime}\right) \mathrm{d} t^{\prime}\right\} \hat{x},
\end{aligned}
$$

where $\tau$ is the relaxation time of the nuclear response. $\tau$ is $2.2 \times 10^{-7} \mathrm{sec}^{9}$ at $44.5^{\circ} \mathrm{C}$, the temperature of our experiment. The induced nonlinear polarization will radiate a wave front $E_{3}(\mathbf{r}, \mathbf{t})$ that is the conjugate of the incident field $E_{4}(\mathbf{r}, t) .{ }^{10}$ The conjugate wave front, after being generated, proceeds to propagate in the opposite direction, retracing the original path of $E_{4}(\mathbf{r}, t)$.

A frequently quoted property of phase conjugation is its ability to compensate for phase distortions. For example, if an incident plane wave passes through a phase-distorting medium, such as a poor-quality optical window or lens, it will acquire an undesirable phase $\exp [i \phi(x, y)]$. The conjugate of this distorted wave will have a phase $\exp [-i \phi(x, y)]$, which, on tracing the original path through the phase-distorting medium, will emerge as a plane wave with the phase aberration exactly canceled. 


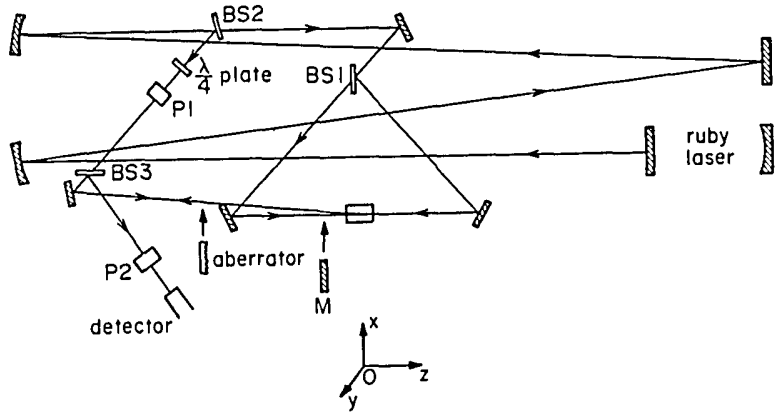

Fig. 1. Experimental setup.

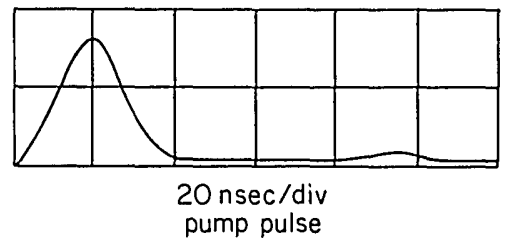

(a)

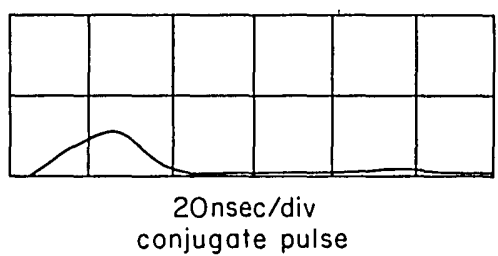

(b)

Fig. 2. The temporal profiles of a typical (a) pump pulse and (b) conjugate pulse.

We note that, if the nonlinear polarization is to be calculated as given in Eq. (6), the fields $E_{1}(\mathbf{r}, t), E_{2}(\mathbf{r}, t)$, and $E_{4}(\mathbf{r}, t)$ will have to be corrected for their attenuation by scattering in the medium. ${ }^{9}$ Furthermore, when the fields are intense optical pulses, as they were in our experiments, the fields have complicated temporal and spatial profiles that are due to the transient self-focusing effect inside the MBBA. ${ }^{2}$

The experimental setup is shown in Fig. 1. The laser source was a $Q$-switched ruby laser operated in a single longitudinal and transverse mode. The linearly polarized ( $s$ polarization) laser pulse had a duration of about $20 \mathrm{nsec}$ and a typical energy of $10 \mathrm{~mJ}$. The intensity of the beam varied transversely as $\exp \left(-r^{2} / w_{0}{ }^{2}\right)$ with a beam radius $w_{0}$ of $0.33 \mathrm{~mm}$. The laser pulse was divided by a beam splitter (BS1) into two beams, which passed through the sample cell in opposite directions to provide the two pump beams, $E_{1}(\mathbf{r}, t)$ and $E_{2}(\mathbf{r}, t)$, required for the four-wave mixing interaction. The field at the plane of the laser output mirror was imaged by two 6 -m radius-of-curvature mirrors onto a plane at the center of the sample cell. The path length between the laser and the cell was therefore $12 \mathrm{~m}$, and this provided a round-trip length of $24 \mathrm{~m}$, or, equivalently, 80-nsec delay to prevent feedback of the pulse to the laser. A small portion of the laser energy was diverted by a beam splitter (BS2) to serve as the signal wave, $E_{4}(\mathbf{r}, t)$. Its polarization was converted by a quarterwave plate and a polarizer ( $\mathrm{P} 1)$ to be orthogonal ( $p$ polarization) to those of the two pump waves. The signal beam entered the cell almost parallel to the pump waves. The angle was limited to about $10 \mathrm{mrad}$ to allow for a large overlap of the beams and hence a large interaction region.

The nonlinear medium was MBBA contained in a 5.7-cm-long quartz cell. The cell was first pumped and then sealed with an atmosphere of $\mathrm{N}_{2}$. The cell was next inserted into a closely fitted copper block with a heating tape wound on the outside. The temperature was monitored and controlled to within $\pm 0.2^{\circ} \mathrm{C}$.

The two $s$-polarized counterpropagating pump waves and the $p$-polarized signal wave induced a nonlinear polarization inside the MBBA that radiated a $p$-polarized conjugate wave. The conjugate beam propagated backward along the path of the signal wave and was directed by a beam splitter (BS3) to a detector. The polarizer P2 in Fig. 1 passed only the $p$-polarized conjugate wave and rejected any $s$-polarized light that was caused by the scattering of the intense pump waves.

A typical pump pulse, shown in Fig. 2(a), has a Gaussian profile with a $1 / e$ width of about $25 \mathrm{nsec}$. The conjugate beam, detected only when all three input pulses were present, is shown in Fig. 2(b) and was found to have a similar pulse width to that of the pump pulses. If the relaxation time of the medium is shorter than the duration of the incident pulses, the conjugate pulse would be shorter than the pump pulses because, according to Eq. (6), it is proportional to the product of three (Gaussian) pulses. ${ }^{11}$ In MBBA, the long relaxation time of the nuclear response integrates out the product $E_{1}(t) E_{4}^{*}(t)$ so, according to Eq. (6), the induced polarization is proportional to that of the pump pulses. A similar situation, but on a much shorter time scale, was reported elsewhere ${ }^{12}$; subpicosecond optical pulses were used to produce conjugate wave fronts in $\mathrm{CS}_{2}$, which has a relaxation time of several picoseconds. The observed conjugate pulse was claimed, however, contrary to our results, to be shorter than the pump pulses. The discrepancy indicates that the electronic contribution to the optical nonlinearity dominates in the subpicosecond time scale.

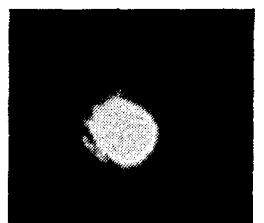

(a)

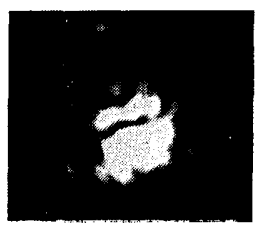

(b)

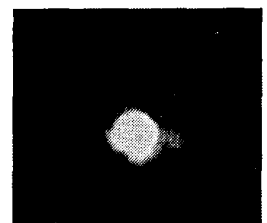

(c)

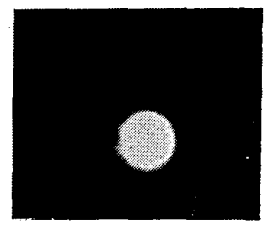

(d)
Fig. 3. The ability of the conjugate beam to correct for distortion (see text): (a) beam reflected from a mirror, (b) beam reflected from a mirror with an aberrator in its path, (c) conjugate beam with the aberrator in its path, (d) conjugate beam. 


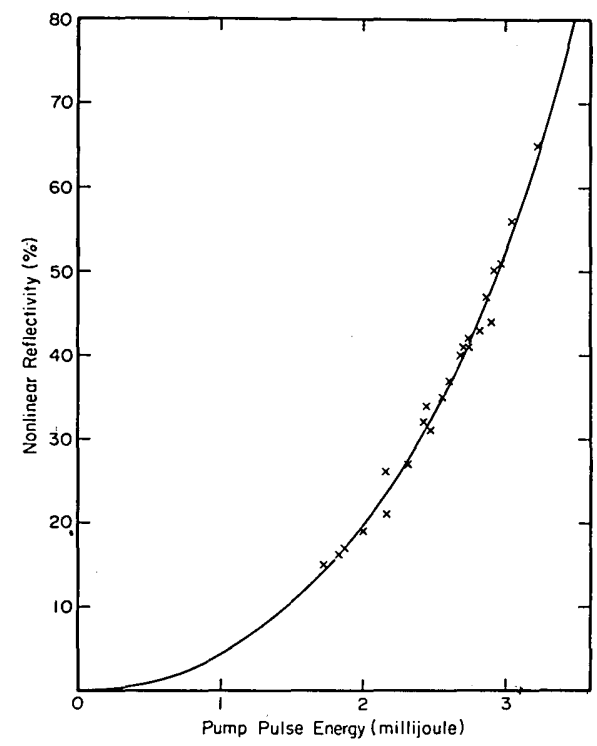

Fig. 4. The nonlinear reflectivity versus the pump-pulse energy.

To verify the conjugate nature of the radiated field and its ability to compensate for phase distortions, the following tests were carried out. First, a mirror $M$ as shown in Fig. 1 was placed next to the sample cell to retroreflect the signal beam, and Fig. 3(a) shows the spatial quality of the beam. Second, a phase aberrator was inserted in the path of the signal beam $E_{4}$, and the picture of the reflected beam from the mirror $M$ was taken. The beam was thus made to pass through the aberrator twice. Figure 3(b) shows such a picture, for which the aberrator was a plece of transparent double-stick Scotch tape. The mirror was then removed to allow the four-wave mixing to take place. Figure 3(c) shows the generated conjugate beam, which passed through the same aberrator before incidence onto the film. Figure $3(\mathrm{~d})$ shows the picture of the conjugate beam with the aberrator removed. Since the conjugate beam had a different intensity from the mirror-reflected signal beam, Figs. 3(a) and 3(b) were taken with an appropriate neutral-density filter in front of the film to simulate the intensity of the conjugate beam. The correction for phase distortion by the conjugate beam becomes evident by comparing Figs. 3(b) and 3(c).

The energy of the conjugate beam was measured and expressed as a percentage of the input-signal energy. The result was plotted in Fig. 4 as a function of the pump-pulse energy. The data points fit very well a curve (solid line in Fig. 4$) \tan ^{2}\left(0.209 \epsilon_{\mathrm{p}}\right)$, where $\epsilon_{p}$ is the pump-pulse energy. This agrees with the functional dependence predicted by the coupled-wave formalism. ${ }^{13}$ We noticed that the nonlinear reflectivity varied with the temperature of the medium, peaking around $55^{\circ} \mathrm{C}$. In particular, the nonlinear effect decreased as the medium was cooled to near the transition point because of the increased scattering loss, in agreement with Shen's result. ${ }^{2}$

The nonlinear coefficient $B$, as deduced from our data, was smaller than what was expected. It was shown by Shen et al. ${ }^{2}$ that the spot size of an intense laser pulse has a complicated profile because of the transient self-focusing effect inside the MBBA; and self-focusing of the laser beam was indeed detected in this experiment. Therefore, good overlapping of the interacting beams was difficult to achieve, giving rise to a weak conjugate signal from which the nonlinear coefficient was deduced. The focusing effect will become more severe if the laser energy is increased, thus making larger nonlinear reflectivity impossible to attain. However, if a circularly polarized pump beam is employed, the self-focusing effect can be reduced. This is because self-focusing of a circularly polarized wave depends on the susceptibility tensor element $\chi_{i j i j}$ $\left(=\chi_{i i j j}\right)$, which is exceedingly small in MBBA. ${ }^{9}$ We have obtained, using this technique with the two pump beams circularly polarized in the opposite sense, a nonlinear reflectivity of $230 \%$ at $4.4 \mathrm{~mJ}$ of pump-beam energy.

In conclusion, we have demonstrated the generation of conjugate wave fronts by degenerate four-wave mixing in the pretransitional state of MBBA.

This research was supported by the U.S. Army Research Office, Durham, North Carolina.

\section{References}

1. D. V. G: L. Narasimha Rao and S. Jayaraman, Appl. Phys. Lett. 23, 539 (1973).

2. G. K. L. Wong and Y. R. Shen, Phys. Rev. Lett. 32, 527 (1974).

3. D. V. G. L. Narasimha Rao and D. K. Agrawal, Phys. Lett. 37A, 383 (1971).

4. J. W. Shelton and Y. R. Shen, Phys. Rev. Lett. 25, 23 (1970).

5. J. W. Shelton and Y. R. Shen, Phys. Rev. Lett. 26, 538 (1971).

6. J. W. Shelton and Y. R. Shen, Phys. Rev. A 5, 1867 (1972).

7. T. W. Stinson III and J. D. Litster, Phys. Rev. Lett. 25, $503(1970)$.

8. G. K. L. Wong and Y. R. Shen, Phys. Rev. Lett. 30, 895 (1973).

9. G. K. L. Wong and Y. R. Shen, Phys. Rev. A 10, 1277 (1974).

10. A. Yariv, IEEE J. Quantum Electron. QE-14, 650 (1978).

11. D. M. Pepper, D. Fekete, and A. Yariv, Appl. Phys. Lett. 33, 41 (1978).

12. D. M. Bloom, C. V. Shank, R. L. Fork, and O. Teschke, "Subpicosecond optical gating and wavefront conjugation by four-wave mixing," in Proceedings of First Topical Meeting on Picosecond Phenomena, C. V. Shank, E. P. Ippen, and S. L. Shapiro, eds. (Springer-Verlag, Berlin, 1978).

13. A. Yariv and D. M. Pepper, Opt. Lett. 1, 16 (1977). 